\title{
Quantifying benefits and risks of vaccinating Australian children aged six months to four years with trivalent inactivated seasonal influenza vaccine in 2010
}

H Kelly (heath.kelly@mh.org.au) ${ }^{1}$, D Carcione ${ }^{2}$, G Dowse ${ }^{2}$, P Effler $^{2}$

1. Victorian Infectious Diseases Reference Laboratory, Melbourne, Australia

2. Communicable Disease Control Directorate, Department of Health Western Australia, Perth, Australia

Citation style for this article:

Kelly H, Carcione D, Dowse G, Effler P. Quantifying benefits and risks of vaccinating Australian children aged six months to four years with trivalent inactivated seasonal influenza vaccine in 2010. Euro Surveill. 2010;15(37):pii=19661. Available online: http://www.eurosurveillance.org/ViewArticle.aspx?Articleld=19661

Article published on 16 September 2010

Australian and New Zealand health authorities identified seasonal trivalent inactivated influenza vaccines manufactured by CSL Biotherapies as the probable cause of increased febrile convulsions in children under five within 24 hours of vaccination and recommended against their use in this age group. We quantified the benefit-risk profile of the CSL vaccines using the number needed to vaccinate and suggest they might have caused two to three hospital admissions due to febrile convulsions for every hospital admission due to influenza prevented.

\section{Introduction}

The recognition of an unexpectedly high number of febrile convulsions (not defined precisely initially) in children aged less than five years within 24 hours of receipt of trivalent inactivated influenza vaccine (TIV) in Australia in April 2010, led to the initial suspension of the childhood vaccination campaign for seasonal influenza in our country [1]. This was followed by more general discussion about the safety of influenza vaccines in children [2].

In 2010, trivalent vaccines from three manufacturers, CSL Biotherapies, Solvay Pharmaceuticals and SanofiPasteur, had been licensed for use in Australian children. All vaccines contained the strains recommended by the World Health Organization (WHO) [3]. The influenza $A\left(\mathrm{H}_{1} \mathrm{~N}_{1}\right)$ virus vaccine component of these vaccines was the 2009 pandemic strain.

The increase in febrile convulsions was first recognised in the state of Western Australia [4]. This was possible because at the beginning of 2008 Western Australia had implemented a vaccine programme aimed at immunising children aged from six months to four years against influenza [5]. This programme acknowledged (i) the importance of children in the spread of influenza, (ii) the high hospitalisation rate due to laboratory-confirmed influenza in this age group and (iii) three childhood deaths associated with influenza in the state in 2007 [5]. Influenza vaccine coverage of $20 \%-30 \%$ had been achieved in this age group for
2008 and 2009 and also in 2010, prior to the suspension of the programme.

Given this background and following concern about the possible risk of febrile convulsions associated with influenza vaccination, the childhood influenza vaccination programme was suspended by the health authorities in Western Australia on 22 April 2010. For the whole of Australia, the precise number of febrile convulsions associated with the administration of TIV was not clear at the time. However, as a precautionary measure, the Australian Department of Health and Ageing recommended against using any formulation of 2010 TIV in young children on 23 April 2010 [1].

At the same time, the Ministry of Health in New Zealand recommended specifically against the use of Fluvax or Fluvax Junior (both manufactured by CSL Biotherapies) in children from six months to four years, but recommended the continued use of other influenza vaccines licensed for children in this age group, specifically Influvac (Solvay Pharmaceuticals) and Vaxigrip (Sanofi-Pasteur) [6], two vaccines that were also licensed in Australia. Recommendations for other age groups remained unchanged. Three months later, on 30 July 2010, the Australian Department of Health and Ageing published revised recommendations indicating it was safe to use either Influvac or Vaxigrip in all children aged from six months to four years but recommended against the use of Fluvax or Fluvax junior in children aged under five years [7].

A timeline of recommendations from health authorities in Australia and New Zealand is shown in Table 1.

Decisions regarding the suspension of an immunisation programme are important because they may have consequences for individuals and a long-term impact on vaccination programmes overall. It is therefore important that these decisions are based on objective assessments of the benefit and risk of all administered vaccines. We present an approach for quantifying the benefit-risk profile for vaccines from 
two manufacturers, CSL Biotherapies and Solvay Pharmaceuticals, in 2010. We briefly compare with data collected in Western Australia on the frequency of febrile convulsions following administration of vaccines from Sanofi-Pasteur and CSL Biotherapies in 2008 and 2009.

\section{Methods}

We compared the benefit-risk profile of vaccination using data for the two vaccines (CSL Biotherapies and Solvay Pharmaceuticals) that had been administered in sufficient numbers in 2010 for meaningful comparisons to be made. To compare benefit and risk, we used hospitalisation data from 2009, obtained from the Department of Health in Western Australia, and adverse event data from 2010, sourced from a detailed investigation of adverse events in Western Australia (unpublished data).

We assessed benefit by estimating the number of children that would have required vaccination to prevent one hospital admission due to laboratory-confirmed influenza. We chose hospitalisation due to influenza of any type or subtype during the 2009 influenza $A\left(\mathrm{H}_{1} \mathrm{~N}_{1}\right)$ pandemic because viral testing for children hospitalised with febrile illnesses was intensive and relatively few children hospitalised due to influenza in 2009 would have remained undiagnosed.

We assessed risk using hospital admissions for febrile convulsions, which were defined by a systematic review of cases with reference to the criteria of the Brighton Collaboration for febrile convulsions following immunisation [8]. Receipt of at least one dose of influenza vaccine in $\mathbf{2 0 1 0}$ was verified from the Australian Childhood Immunisation Register [9] and direct questioning of parents or guardians.

\section{Quantifying benefit}

To quantify benefit, we calculated the number of children that would need to be vaccinated to prevent one hospital admission due to influenza, that is, the number needed to vaccinate (NNV). The NNV is calculated as the reciprocal of the absolute risk reduction. The absolute risk reduction is calculated as the product of the absolute risk in the unexposed (in this

\section{TABLE 1}

Timeline of decisions communicated by health authorities in Australia and New Zealand following recognition of febrile convulsions in children under five years occurring within 24 hours of receipt of trivalent inactivated vaccine, 2010

\begin{tabular}{|c|c|}
\hline Date (2010) & Decision \\
\hline 22 April & $\begin{array}{l}\text { The Health Department of Western Australia suspends its influenza vaccination programme for children under five years } \\
\text { of age because of concern about an unexpected number of febrile convulsions in children within } 24 \text { hours of receipt of the } \\
\text { seasonal vaccine. }\end{array}$ \\
\hline 23 April & $\begin{array}{l}\text { The Australian Department of Health and Ageing suspends the influenza vaccination programme at national level for children } \\
\text { under five years of age. }\end{array}$ \\
\hline 27 April & $\begin{array}{l}\text { The New Zealand Ministry of Health writes to general practitioners, recommending them not to use influenza vaccines } \\
\text { manufactured by CSL Biotherapies (Fluvax and Fluvax Junior), but to continue using licensed vaccines from other } \\
\text { manufacturers for children under five years of age. }\end{array}$ \\
\hline 1 June & $\begin{array}{l}\text { The Australian Department of Health and Ageing recommends influenza vaccination can be resumed for children at risk of a } \\
\text { severe outcome of influenza. It suggests the CSL vaccines are most likely responsible for the unexpected number of febrile } \\
\text { convulsions. Influenza vaccines, including CSL products, can be administered on a case by case basis. }\end{array}$ \\
\hline 8 July & $\begin{array}{l}\text { The New Zealand Ministry of Health reiterates its previous advice to general practitioners regarding the use of influenza } \\
\text { vaccines for children under five years of age. }\end{array}$ \\
\hline 30 July & $\begin{array}{l}\text { The Australian Department of Health and Ageing recommends that vaccination of healthy children under five years of age } \\
\text { can resume, but not with CSL vaccines. The report states that 'continued close monitoring of side effects with this year's } \\
\text { seasonal flu vaccine in children under five years of age has shown that the higher than usual occurrence of fever and febrile } \\
\text { convulsions appears to be confined to the vaccine Fluvax, manufactured by CSL.' }\end{array}$ \\
\hline
\end{tabular}

\section{TABLE 2}

The number needed to vaccinate to prevent one hospital admission for laboratory-confirmed influenza, all subtypes, and the risk of hospital admission for febrile convulsion following receipt of trivalent inactivated influenza vaccine ${ }^{\text {a }}$ by year and vaccine manufacturer

\begin{tabular}{|c|c|c|c|c|c|c|}
\hline Year & Manufacturer & $\begin{array}{c}\text { NNV to prevent one } \\
\text { hospital admission } \\
\text { for influenza (2009) }\end{array}$ & $\begin{array}{c}\text { Risk of hospital } \\
\text { admission } \\
\text { for febrile } \\
\text { convulsion }\end{array}$ & $\begin{array}{c}\text { Number of hospital } \\
\text { admissions for febrile } \\
\text { convulsions following } \\
\text { vaccination of NNV }\end{array}$ & $\begin{array}{c}\text { Risk of hospital admission } \\
\text { for febrile convulsion } \\
\text { (upper limit 95\% Cl) }\end{array}$ & $\begin{array}{c}\text { Number of hospital } \\
\text { admissions for febrile } \\
\text { convulsions } \\
\text { (upper limit 95\% Cl) }\end{array}$ \\
\hline \multirow{2}{*}{2010} & CSL & 1,852 & 0.0013 & 2.4 & 0.0017 & 3.1 \\
\cline { 2 - 7 } & Solvay & 1,852 & 0 & 0 & 0.0003 & 0.6 \\
\hline 2009 & CSL/Sanofi & 1,852 & 0 & 0 & Not quantified & Not quantified \\
\hline 2008 & CSL/Sanofi & 1,852 & 0.00001 & 0 & Not quantified & Not quantified \\
\hline
\end{tabular}

a For 2010, the influenza $\mathrm{A}\left(\mathrm{H}_{1} \mathrm{~N}_{1}\right)$ virus vaccine component was the 2009 pandemic strain as recommended by the World Health Organization.

${ }^{b}$ Calculated as risk of febrile convulsion following receipt of TIV $\mathrm{x}$ risk of hospitalisation following febrile convulsion.

$\mathrm{Cl}$ : confidence interval; NNV: number needed to vaccinate. 
case the unvaccinated) and the relative risk reduction. Vaccine effectiveness (VE) is the standard expression of the relative risk reduction for vaccine preventable diseases [10]. Hence, the NNV to prevent one hospitalisation $=1 /$ (hospitalisation rate in the unvaccinated $x$ VE). We therefore needed to estimate two parameters: the hospitalisation rate in the unvaccinated and the VE.

To estimate the hospitalisation rate in the unvaccinated, we ascertained the vaccine status of children admitted to hospital for influenza in 2009, thus allowing us to calculate the number of these children who were not vaccinated. We then estimated vaccine coverage for influenza vaccine in 2009 for children aged between six months and four years from vaccine usage reported by immunisation providers state-wide. This allowed us to estimate the size of the unvaccinated population in this age group $=(1-$ the proportion vaccinated) $x$ (the population in that age group). The number of unvaccinated children admitted to hospital divided by the estimated number of unvaccinated children in the population gave the hospitalisation rate in the unvaccinated.

The VE for prevention of influenza infection in children aged between 2 and 16 years has been estimated in a systematic review as $59 \%$ (95\% confidence interval (Cl): 41 to 71 ) [11]. This estimate is supported by findings from a study of children aged o to 4 years in Western Australia in 2008, in which the VE was 68\% (95\% Cl: 26 to 86 ) [5]. The study found no significant difference in VE by age group (less than two years compared with two to four years) for children with an influenza-like illness proven to be caused by influenza [5]. We assumed the VE for TIV in 2010, the year for which we calculated the benefit-risk profile, would have been similar to previous estimates of VE when vaccine and circulating strains matched. This VE estimate should be appropriate for the TIV for 2010 , as vaccine manufacturing processes were the same in 2010 as for previous years. We therefore used a VE of $60 \%$ for all children aged six months to four years in this analysis.

\section{Quantifying risk}

To quantify risk, we calculated the number of hospitalisations that could be attributed to adverse events following vaccination of the NNV to prevent one hospital admission. We used vaccine-specific rates of febrile convulsions determined during the detailed investigation into suspected adverse outcomes in 2010 in Western Australia (unpublished). We compared these with vaccine-specific rates of febrile convulsions in 2008 and 2009 , determined by passive surveillance in both years but augmented by active questioning of parents/guardians of children admitted to hospital in 2009. We quantified the risk of admission to hospital for a febrile convulsion following receipt of TIV as the product of the NNV, the absolute risk of a febrile convulsion and the risk of hospital admission following a febrile convulsion.

\section{Results}

Quantifying benefit: the NNV to prevent one hospitalisation due to influenza

The number of children living in Western Australia aged between six months and four years in 2009 was approximately 130,000 of whom $30.3 \%$ were estimated to have received at least one dose of TIV in that year. The number of unvaccinated children was therefore 90,610 .

In that year, 432 cases of influenza were notified in children in this age range, of whom $383(89 \%)$ were infected with 2009 pandemic influenza $A\left(\mathrm{H}_{1} \mathrm{~N}_{1}\right)$ and $49(11 \%)$ were infected with seasonal influenza strains or influenza A not subtyped. One hundred and twenty children were hospitalised for any type of influenza, of whom 74 were unvaccinated. Vaccine status was unknown for a further 13 children. We allocated the children with unknown vaccine status to one of the two known groups in the same proportion as those whose status was known, giving an estimated total of 82 unvaccinated children admitted to hospital. The hospitalisation rate in unvaccinated children aged six months to four years was therefore 90 per 100,000 .

To prevent one hospitalisation due to any strain of circulating influenza in 2009 would have required the vaccination of 1,852 children (1/[90/1000,00 x 0.6]), the NNV.

Quantifying risk: hospital admission for febrile convulsions following receipt of TIV Prior to cessation of the immunisation programme in April 2010, more than 10,000 doses of Fluvax or Fluvax Junior and more than 3,300 doses of Influvac had been administered to children aged six months to four years in Western Australia in 2010 [4]. Detailed follow up investigation of adverse events following immunisation identified 56 children with febrile convulsions that occurred within 24 hours of receipt of TIV between 8 March and 25 April 2010, of whom 19 (34\%) required overnight hospital admission. All 55 cases with information on vaccine formulation had received Fluvax or Fluvax Junior. The estimated risk of a febrile convulsion following receipt of Fluvax or Fluvax Junior was $0.39 \%$, compared to $0 \%$ for Influvac (unpublished data).

Applying these estimates of risk to the NNV to prevent one hospitalisation due to influenza indicates that vaccination with Fluvax or Fluvax Junior would have been likely to have caused seven febrile convulsions $(1,852$ $x$ 0.0039), with two to three of these children requiring hospitalisation ( $34 \%$ of seven children). Based on the data available, Influvac would have caused no febrile convulsions and no hospital admissions.

The upper limit of the $95 \% \mathrm{Cl}$ for Influvac estimated a febrile convulsion risk of $0.08 \%$ (unpublished data). If the real risk were this high, Influvac may have caused one to two febrile convulsions, and zero or one hospital admission, in preventing one hospitalisation due to laboratory-confirmed influenza. The corresponding risk of febrile convulsions for the Fluvax products (that 
is, estimating risk as the upper limit of the $95 \% \mathrm{Cl}=$ $0.51 \%$, unpublished data) would have seen nine febrile convulsions attributed to these vaccines, causing three hospital admissions for febrile convulsions for each hospital admission for influenza that was prevented.

Using routine passive surveillance in 2008 and 2009, plus direct enquiry to parents or guardians of children admitted to hospital with laboratory-confirmed influenza in 2009, we estimated the risk of a febrile convulsion following receipt of any seasonal TIV to have been $0.003 \%$ in 2008 and zero in 2009. In those two years, these vaccines would have caused no hospital admissions due to febrile convulsions for each hospital admission due to influenza that was prevented (Table 2).

\section{Discussion and conclusion}

We have demonstrated a method that can be used to quantify the benefit and risk of vaccinating children aged six months to four years against influenza. In 2009 , with relatively high levels of hospital admission, it would have been necessary to have vaccinated about 1,850 West Australian children with a vaccine that was $60 \%$ effective to have prevented one hospital admission due to laboratory-confirmed influenza.

If the hospitalisation rate for influenza in 2010 was the same as that in 2009 , we estimated that vaccination with Fluvax or Fluvax Junior in 2010 may have caused two to three hospital admissions due to febrile convulsions for every hospital admission due to influenza prevented. Although the influenza season is not yet over in Western Australia, current data indicate that influenza virus circulation has been considerably lower in 2010 than in 2009 [12] and fewer children will have been hospitalised for influenza in 2010 than were hospitalised in 2009. This implies that the NNV to prevent one hospital admission in 2010 would be higher than in 2009.

We did not see this same risk profile with another TIV licensed for this age group in 2010, or with the vaccines manufactured by CSL Biotherapies in 2008 or 2009, despite similarly high vaccine coverage in these two years in Western Australia. Our results therefore indicate there is no excess risk over benefit with a childhood influenza vaccination programme in general. The problem identified in 2010 was related to a vaccine produced by a single manufacturer. Investigations published to date have failed to find a cause for the problem [13].

A comprehensive approach to this analysis would involve assessment of other outcomes due to laboratory-confirmed influenza and a detailed sensitivity analysis. Moreover, we have chosen to quantify the NNV using all children aged six months to four years. The benefit-risk profile would be improved if only children who were at increased risk of hospitalisation following influenza infection were targeted for vaccination.
However, we have shown that a good past benefit-risk profile for a vaccine may not guarantee a favourable profile in future years. This highlights once again the importance of continued and comprehensive safety monitoring of influenza vaccines post-marketing.

\section{Acknowledgements}

We acknowledge the contributions of many staff in the Department of Health in Western Australia to the investigation of adverse events in children following influenza vaccination in 2010.

\section{References}

1. Australian Government. Department of Health and Ageing. Seasonal Flu Vaccine and young children. 23 April 2010. Media Release. Available from: http://www.health.gov.au/internet/ main/publishing.nsf/Content/mr-yr10-dept-dept230410.htm

2. Collignon $P$, Doshi $P$, Jefferson $T$. Child influenza vaccination. Ramifications of adverse events in children in Australia. BMJ. 2010;340:C2994.

3. World Health Organization (WHO). Recommended composition of influenza virus vaccines for use in the 2010 southern hemisphere influenza season. WHO; Revised 8 Oct 2009. Available from: http://www.who.int/csr/disease/ influenza/200909 Recommendation.pdf

4. Government of Western Australia. Department of Health. Ministerial Review into the Public Health Response into the Adverse Events to the Seasonal Influenza Vaccine. July 2010. Final Report to the Minister for Health. [Accessed 16 Aug 2010]. Available from: http://www.health.wa.gov.au/publications/ documents/Stokes_Report.pdf

5. Kelly H, Jacoby P, Dixon G, Moore HC, Carcione D, Williams $S$, et al. Vaccine effectiveness against laboratory-confirmed influenza in healthy young children: a case control study. Pediatric Inf Dis. Forthcoming 2010.

6. New Zealand Ministry of Health. Letter to General Practitioners, Practice Nurses, Practice Managers and Health Professionals. 26 April 2010. [Accessed 14 Jul 2010]. Available from: http://www.moh.govt.nz/moh.nsf/pagesmh/9164/\$File/ gp-fluvax-fax-apr2010.doc

7. Department of Health and Ageing. Seasonal flu vaccination for young children can be resumed. Updated advice from the Chief Medical Officer. [Accessed 9 Sep 2010]. Available from: http:// www.health.gov.au/internet/main/publishing.nsf/Content/mryr10-dept-dept300710.htm

8. Bonhoeffer J, Menkes J, Gold MS, de Souza-Brito G, Fisher MC, Halsey N, et al. Brighton Collaboration Seizure Working Group. Generalized convulsive seizure as an adverse event following immunization: case definition and guidelines for data collection, analysis, and presentation. Vaccine 2004; 22(5-6):557-62.

9. Australian Government. Medicare Australia. Australian Childhood Immunisation Register. Available at http://www. medicareaustralia.gov.au/public/services/acir/index.jsp

10. Kelly H, Attia J, Andrews R, Heller RF. The number needed to vaccinate (NNV) and population extensions of the NNV: comparison of influenza and pneumococcal vaccine programmes for people aged 65 years and over. Vaccine 2004;22(17-18):2192-8.

11. Smith S, Demicheli V, Di Pietrantonj C, Harnden AR, Jefferson T, Matheson NJ, et al. Vaccines for preventing influenza in healthy children. Cochrane Database Syst Rev. 2006;(1):CDo04879.

12. Australian Government. Department of Health and Ageing. Australian Influenza Surveillance Report. No. 35, 2010. Reporting period 28 August-3 September 2010. Available from: http://www.healthemergency.gov.au/internet/ healthemergency/publishing.nsf/Content/ozflu2010-jul-seppdf-cnt.htm/\$File/ozflu-n035-2010.pdf

13. Australian Government. Department of Health and Ageing. Therapeutic Goods Administration. Investigation into febrile reactions in young children following 2010 seasonal trivalent influenza vaccination. 2 Jul 2010. Available from: http://www. tga.gov.au/alerts/medicines/fluvaccine-report100702.htm 